\title{
FRAILTY MODELS BASED ON LÉVY PROCESSES
}

\author{
Abstract \\ Generalizing the standard frailty models of survival analysis, we propose to \\ model frailty as a weighted Lévy process. Hence, the frailty of an individual \\ is not a given quantity, but develops over time. Formulae for the population \\ hazard and survival functions are derived. The power variance function (PVF) \\ Lévy process is a prominent example. In many cases, notably for compound \\ Poisson processes, quasi-stationary distributions of survivors may arise. Quasi- \\ stationarity implies limiting population hazard rates that are constant, in spite \\ of the continual increase of the individual hazards. A brief discussion is given \\ of the biological relevance of this finding. \\ Keywords: Frailty; Quasi-stationary; compound Poisson process; cumulative \\ damage process; Lévy process; hazard process; PVF distribution \\ HÅKON K. GJESSING, ${ }^{*}$ University of Oslo and Norwegian Institute of Public Health \\ ODD O. AALEN, ${ }^{* *}$ University of Oslo \\ NILS LID HJORT, ${ }^{* * *}$ University of Oslo
}

AMS 2000 Subject Classification: Primary 62N01

Secondary $60 \mathrm{G} 51 ; 60 \mathrm{~B} 10$

\section{Introduction}

The hazard rate is a theoretical and descriptive tool which plays a fundamental role in survival and event history analysis. Basic methodologies in these fields, like the counting process approach and the Cox model, focus on hazard rates. The purpose of the hazard rate is to measure, locally, the risk of an event given the non-occurrence of the event up to that time. It is an intuitive and attractive concept, and is also closely related to a concept like incidence rate in epidemiology. In a probabilistic context the hazard rate is a special case of an intensity process, or the derivative of a Doob-Meyer compensator, see [6].

In practice, the hazard rate must be estimated from data, based on the observation of a number of individuals over time. One is then faced with the fact that individuals will be dissimilar. In a sense one can say that each individual will have his own hazard rate, and what is estimated on the basis of a number of individuals is some sort of average. It is well known that the average hazard rate may be entirely different from those of the individuals, however. Even when all individuals have hazard rates with the same functional form, but different levels determined by varying proportionality

\footnotetext{
* Postal address: Norwegian Institute of Public Health, P.O.Box 4404, Nydalen, N-0403 Oslo, Norway. Hakon.Gjessing@fhi.no

** Postal address: Section of Medical Statistics, University of Oslo, P.O. Box 1122 Blindern, N-0317 Oslo, Norway. O.O.Aalen@basalmed.uio.no

*** Postal address: Department of Mathematics, University of Oslo, P.O. Box 1053 Blindern, N-0316 Oslo, Norway. Nils@math.uio.no
} 
constants, the estimated hazard rate will have an entirely different shape from that of the individuals. This is pointed out in frailty theory, see [13].

\subsection{Biological interpretations of hazard rate}

In the biological literature one may find attempts at interpreting shapes of hazard rates and drawing biological conclusions from these. In a paper in Nature, Clarke et al. [9] discuss the development of diseases involving neuronal degeneration (e.g. Parkinson's disease). In such diseases the onset of clinical symptoms may be delayed for years or decades after the premature neuronal death has started. There has been a discussion of whether this delay reflects a cumulative damage process, or whether the death of a neuron is basically a random event, the likelihood of which is constant over time. Presumably, the first hypothesis would lead to increasing hazard of cell death, while the second one would give a constant hazard. The cumulative damage model implies a progressive process in the organism whereby previous cell death creates damage that increases the likelihood of new cell death. The random event model, also called a "one-hit" model, implies that there has been damage at one specific time, e.g. a mutation, which then increases the likelihood of cell death to a new fixed level.

For a number of diseases and animal models, Clarke et al. study the survival of neurons. They find that the hazard rate is generally constant, or sometimes decreasing, but not increasing. From this they draw the conclusion that the cumulative damage model is incorrect ant that the one-hit random model is the true one. In a more recent paper, Clarke et al. [10] give a further discussion of their hypothesis. A similar study for a type of mutant mice is presented by Triarhou [19], with the same conclusion of a constant rate of cell death.

Another setting where biological interpretations of hazard rates have been presented, is in the understanding of sleep. Lo et al. [16] study the changes between sleep and wake states throughout the night. They find that the duration of sleep periods have more or less an exponential distribution, while the duration of wake states has a distribution with a decreasing hazard rate. This is interpreted within a stochastic process context, but basically the implication is that sleep represents a random walk with no drift.

What is common for these papers is that they draw biological conclusions from the shapes of hazard rates. It is then important to understand how very different underlying models may lead to similar hazard rates. In particular, an approximately constant hazard rate will be a common phenomenon for many models due to convergence to quasi-stationarity. It therefore appears difficult to draw conclusions about the underlying process with any degree of certainty.

An added difficulty comes from the possibility of frailty variation, that is, heterogeneity in risk between different units, those units being either individual cells or organisms. Hence the development of risk at the level of an individual neuron, say, is very hard to deduce.

This criticism does not necessarily imply that the conclusions in the mentioned papers are wrong. But it points to the necessity of understanding the hazard rate and how its various shapes can arise. 


\subsection{A model for random hazard processes}

The standard approach for combining a model for the hazard rate with an allowance for individual heterogeneity in risk is the frailty model

$$
\mu_{i}(t)=Z_{i} \lambda(t) \exp \left(\boldsymbol{\beta}^{T} \mathbf{x}_{i}\right) .
$$

The deterministic hazard rate $\lambda(t)$ is estimated (parametrically or non-parametrically) from data. The exponential term allows the parameter vector $\boldsymbol{\beta}$ to be estimated, giving the effect of the covariate vector $\mathbf{x}_{i}$ on the hazard. The individual frailty $Z_{i}$ is drawn from a distribution of non-negative random variables. The subscript $i$ indicates that a new realization of $Z$ is drawn for each individual. For more information on standard frailty models and many extensions, see [13] and [2]. The simplifying assumptions made in a standard frailty model enables one to estimate the true underlying hazard of individuals, which is easier when multivariate survival data are available, as compared to univariate data. However, there are two important aspects of the simplifying assumptions. First, it is assumed that all individuals have proportional hazards. This is obviously an assumption of mathematical convenience, although the theory developing from it also seems to yield useful insights into biological phenomena. Second, the individual frailty $Z_{i}$ is determined at time zero and follows the individual throughout its entire life, resulting in the individual risk later in life to be perfectly correlated with that at the beginning. It is clearly of interest to develop more flexible models.

A general view of the individual variation in hazard rates would be to regard the hazard of an individual as a stochastic process. This would give individual flexibility. However, since what is observable is still the average hazard rate, there should be a tractable mathematical connection between the individual hazard rates and the average one. One approach, which has been tried with some success by Yashin and others, is to define the hazard rate as a diffusion process by means of a stochastic differential equation [20]. It is not unnatural to think that the risk of an individual may develop as some kind of diffusion. By introducing Ornstein-Uhlenbeck type processes one may model the fact that many biological parameters tend to stabilize around certain values, or what is called homeostasis.

Here we shall attempt a different approach, namely one based on Lévy processes. This has been suggested before and developed to some extent by Kebir [14], but we shall here give a number of additional results. Like diffusion processes, hazard rates driven by Lévy processes also yield some degree of tractability. It is possible to get explicit formulae for the relationship between individual and average hazard, one may calculate the frailty and hazard of survivors, and so on. A basic difference from diffusion processes is the jump nature of the non-negative Lévy processes applied here. However, one could well imagine that individual hazard may increase in jumps, for instance by the onset of an acute disease.

In the present paper we shall not go into statistical estimation. The object is to study a mathematically tractable framework. One type of results we shall focus on is the occurrence of quasi-stationarity in some models. This means that even though individuals are constantly leaving the risk set, the distribution of the hazard of the survivors may still converge to a limit. An effect of this will be that the average hazard converges towards a constant level, which is sometimes seen in practice. In first-passage time models quasi-stationarity is important in understanding the shape of the hazard rate [3]. 
The extension of frailty theory proposed here is hoped to have some biological relevance. When looking at the risk of disease it will usually be the case that some of the risk is acquired already at birth, for instance by the genetic makeup of the individual. Then additional risk accumulates throughout life dependent on life style, various life circumstances and accidental events. Also, important biological parameters, like blood pressure and cholesterol, typically increase throughout life. If the focus is on the development of some disease, then this may depend on observable marker processes, like the CD4 counts in HIV infection. Such marker processes yield stochastic hazards [18]. There is presently a development in survival analysis towards joint modelling of survival on the one hand and marker or covariate processes on the other hand. Mathematical frameworks, like the one we present here, may be useful for such developments.

We shall consider here frailty distributions defined by a non-negative Lévy process, which in our paper is taken to mean a process with non-negative, independent, timehomogeneous increments, i.e. a subordinator. The Laplace transform of such a process $Z=\{Z(t): t \geq 0\}$ at time $t$ is given by the Lévy-Khintchine formula

$$
L(c ; t)=E \exp \{-c Z(t)\}=\exp \{-t \Phi(c)\}
$$

where $c \geq 0$ is the argument of the Laplace transform. The function $\Phi(c)$ is called the Laplace exponent of the Lévy process. The family of Lévy processes contains a number of important special cases, like compound Poisson processes, gamma processes, stable processes, etc. In fact, all non-negative Lévy processes are limits of compound Poisson processes. For background material on Lévy processes see e.g. [8].

When $Z(t)$ is a subordinator, it is natural to associate frailty with such a process, since frailty of an individual frequently can be thought of as increasing over time. We would want to extend this to more general situations, however. To consider processes with a varying "rate", define the non-negative deterministic rate function $r(t)$ with integral $R(t)=\int_{0}^{t} r(u) d u$, and let $Z(R(t))$ be the time-transformed subordinator. Conditional on $Z$, we define our basic hazard rate processes $h$ as

$$
h(t)=\lambda(t) \int_{0}^{t} a(u, t-u) d Z(R(u)) .
$$

This process will be the starting point for all models considered in our paper. The function $a(u, s)$ is a weight function determining the extent to which the effect of previous jumps in $Z(t)$ influence the hazard at time $t$. By having two arguments in $a(u, s)$, one allows for the weight depending on two time scales, namely the time scale of the stochastic process (first argument), and time as measured in distance from the current time (second argument). The deterministic function $\lambda$ determines the "base" level of the hazard, although it could just as well be absorbed in $a$. Since most frailty distributions of the classical frailty models are distributions of Lévy processes, the general formulation here incorporates most of these classical models, as will be seen below. Notice that, like in the standard frailty model (1), each individual in the population will have its own realization of the underlying process $Z$ and thus of the hazard process $h$, although we will suppress the subscript $i$.

An introduction to models similar to the ones considered here, is given by Kebir in [14]. An overview over hazard processes developing under randomness is given by Singpurwalla [17]. Some models of frailty using Lévy processes are discussed in [4] and [11]. Our results extend and complement those of Kebir. 
We will start by giving a short review of Lévy processes with some examples in Section 2. In Section 3 some general formulae for the population hazard and survival are presented, together with a collection of special cases to illustrate the results. Section 4 discusses the distribution of survivors in the model, in particular the variance of the hazard distribution at time $t$. In Section 5 we study the long-run distribution of the hazard and frailty, which frequently exhibits quasi-stationarity. Some of the special cases from Section 3 are studied in this context. Examples of explicit formulae using the power variance function (PVF) processes, introduced in Subsection 2.5, are discussed in Section 6. Section 7 demonstrates how our framework is a natural formulation of models with individual random perturbations of a parametric hazard function.

\section{Lévy processes and subordinators}

The full distribution of a non-negative Lévy process, i.e. a subordinator $Z$, is determined by its Laplace exponent $\Phi$, defined in (2). For this reason many general results on subordinators are derived in terms of $\Phi$. The function $\Phi(\cdot)$ is increasing and concave, its derivative $\Phi^{\prime}$ decreasing. We have the general representation

$$
\Phi(c)=đ c+\int_{(0, \infty)}\left(1-e^{-c x}\right) \Pi(d x),
$$

where đ is called the drift coefficient since at time $t$ the distribution of $Z(t)$ is shifted to the right by an amount $t$ d. The measure $\Pi(d x)$ has support on $(0, \infty)$ and satisfies the condition $\int_{(0, \infty)}(1 \wedge x) \Pi(d x)<\infty$. It is called the Lévy measure of the process $Z$. Notice that when $\int_{(0, \infty)} \Pi(d x)=\rho<\infty$, i.e. when $(1 / \rho) \Pi$ is a probability measure on $(0, \infty)$, we can write

$$
\Phi(c)=đ c+\rho\left(1-L_{0}(c)\right)
$$

where $L_{0}(c)$ is the Laplace transform of $(1 / \rho) \Pi$. For the derivatives we have

$$
\begin{aligned}
& \Phi^{\prime}(c)=\mathrm{d}+\int_{(0, \infty)} x e^{-c x} \Pi(d x), \\
& \Phi^{\prime \prime}(c)=-\int_{(0, \infty)} x^{2} e^{-c x} \Pi(d x),
\end{aligned}
$$

which both exist for $c>0$. Clearly, $\lim _{c \rightarrow \infty} \Phi^{\prime}(c)=$ đ. When $c=0$ we obtain the relationships

$$
\begin{aligned}
E Z(t) & =\Phi^{\prime}(0) t=\left(\mathrm{d}+\int_{(0, \infty)} x \Pi(d x)\right) t, \\
\operatorname{Var} Z(t) & =-\Phi^{\prime \prime}(0) t=\int_{(0, \infty)} x^{2} \Pi(d x) t
\end{aligned}
$$

which in some cases will be infinite.

We will present a number of examples of Lévy processes.

\subsection{Standard compound Poisson process}

Distributions generated by a compound Poisson process have previously been used as frailty models, see e.g. [1] and [5]. They are important examples in the present 
framework of Lévy processes. A compound Poisson process is constructed as follows. A Poisson process of rate $\rho$ is running on time scale $t$, and to each jump there is a gamma random variable, independent of the Poisson process, with shape parameter $n>0$ and scale parameter $\nu>0$. The compound Poisson process is the sum of the gamma random variables up to time $t$. The Laplace transform of the distribution of the process at time $t$ is given by $L(c ; t)=\exp \left\{-t \rho\left[1-(\nu /(\nu+c))^{n}\right]\right\}$. Hence,

$$
\Phi(c)=\rho\left\{1-L_{0}(c)\right\}
$$

where $L_{0}(c)=(\nu /(\nu+c))^{n}$ is the Laplace transform of the Gamma distribution of the jumps, and

$$
\Pi(d x)=\rho \frac{\nu^{n}}{\Gamma(n)} x^{n-1} e^{-\nu x} d x
$$

\subsection{Compound Poisson process with general jump distribution}

Let the gamma variables be replaced by positive random variables $X$ with distribution $\mu_{X}(d x)$ and Laplace transform $L_{0}(c)=E \exp (-c X)$. The Laplace transform of the process now takes the form $L(c ; t)=\exp \left\{-\rho t+\rho t L_{0}(c)\right\}$, giving

$$
\Phi(c)=\rho\left\{1-L_{0}(c)\right\} \text {. }
$$

The Lévy measure $\Pi(d x)=\rho \mu_{X}(d x)$ is proportional to the jump distribution $\mu_{X}$.

It is true in general that $\Phi$ is bounded if and only if $Z$ is compound Poisson. This is proved for the characteristic exponent in [8], but the argument is easily adapted to $\Phi$.

\subsection{Gamma processes}

Now let the value of the process at time $t$ be gamma distributed with shape parameter $\rho t$ and scale parameter $\nu$. Then $L(c ; t)=(\nu /(\nu+c))^{\rho t}=\exp \{-\rho t[\log (\nu+c)-$ $\log \nu]\}$, so that $\Phi(c)=\rho\{\log (\nu+c)-\log \nu\}$. Here $\Pi(d x)=\rho e^{-\nu x} / x d x$. This can be considered a borderline case of the standard compound Poisson process in 2.1 when $n=0$ and $\rho \rightarrow \infty$ in such a way that $n \rho$ converges to a positive constant.

\subsection{Stable processes}

Stable frailty distributions have been applied successfully by Hougaard ([12], [13]) who has pointed out that they may preserve proportional hazards. The Laplace transform takes the form $L(c ; t)=\exp \left(-t \alpha c^{\beta}\right)$, where $\beta$ is a parameter in $(0,1)$. Hence $\Phi(c)=\alpha c^{\beta}$, with Lévy measure

$$
\Pi(d x)=\frac{\alpha \beta}{\Gamma(1-\beta)} x^{-1-\beta} d x
$$

\subsection{PVF processes}

The PVF (power variance function) distributions constitute a general class of distributions studied, for instance, in [13]. A class of Lévy processes may be defined from the PVF distributions by letting

$$
\Phi_{\mathrm{PVF}}(c ; \rho, \nu, n)=\rho\left\{1-\left(\frac{\nu}{\nu+c}\right)^{n}\right\}
$$


with $\nu>0, n>-1$ and $n \rho>0$. This is a direct extension of the standard compound Poisson process defined in (4) above. The Lévy measure is

$$
\Pi(d x)=n \rho \frac{\nu^{n}}{\Gamma(n+1)} x^{n-1} e^{-\nu x} d x .
$$

When $n>0$ this is the standard compound Poisson. For $n=0$ we recognize the gamma process as a borderline case. When $0>n>-1$ then $\Pi([0, \infty))=\infty$, so the process is no longer compound Poisson, but the special case of $n=-1 / 2$ is the inverse Gaussian process, and when $\nu \rightarrow 0$ and $\rho \rightarrow \infty$ in such a way that $\rho \nu^{n}$ converges to a positive value, the result it is a stable process. Section 6 is devoted entirely to examples using PVF processes.

\section{Results for general and special frailty processes}

In a population with frailty, assume the hazard for an individual at time $t$ is $h(t)$, a suitable non-negative stochastic process. Let $T$ be the random time at which an event occurs for an individual. Let $\mathbb{I}(T>t)=1$ if $T>t, 0$ otherwise. Clearly, the population survival $S(t)=P(T>t)$ is

$$
S(t)=E[\mathbb{I}(T>t)]=E[E[\mathbb{I}(T>t) \mid h]]=E \exp \left(-\int_{0}^{t} h(s) d s\right) .
$$

Conditional on $h$, we have $P(T>t \mid h)=\int_{t}^{\infty} h(s) P(T>s \mid h) d s$. Taking expectations on both sides,

$$
P(T>t)=\int_{t}^{\infty} E[h(s) E[\mathbb{I}(T>s) \mid h]] d s=\int_{t}^{\infty} E[h(s) \mid T>s] P(T>s) d s,
$$

thus the population survival $S(t)$ has the corresponding population hazard

$$
\mu(t)=E[h(t) \mid T>t],
$$

see, for instance, [20].

If the distribution of $h(t)$, conditional on $T>t$, converges to a non-degenerate distribution when $t \rightarrow \infty$, we refer to this as a quasi-stationary distribution for the hazard of the survivors. Similarly, in the model (3) we can consider a quasi-stationary distribution for the underlying frailty process $Z(t)$.

From now on, $h$ will mean the frailty formulation (3).

Condition 1. To assure the existence of a population hazard $\mu(t)$ for all $t>0$, we assume that $\lambda$ and $r$ are bounded on compact subsets of $[0, \infty)$, and that $\int_{0}^{l} a(t, v) d v<$ $\infty$ for all $l, t \geq 0$.

\subsection{Population versus individual survival}

If we follow the hazard of an individual over time, regardless of whether an event has occurred or not, then

$$
\begin{aligned}
E[h(t)] & =\lambda(t) E[Z(1)] \int_{0}^{t} a(u, t-u) r(u) d u, \\
\operatorname{Var}[h(t)] & =\lambda(t)^{2} \operatorname{Var}[Z(1)] \int_{0}^{t} a(u, t-u)^{2} r(u) d u .
\end{aligned}
$$


We have seen above that to study the population hazard we need to consider the corresponding values conditional on $T>t$.

Theorem 1. Under the model (3) for the hazard, we have the following expressions for the population survival and hazard functions:

$$
\begin{aligned}
& S(t)=\exp \left(-\int_{0}^{t} \Phi(b(u, t)) r(u) d u\right), \\
& \mu(t)=\lambda(t) \int_{0}^{t} \Phi^{\prime}(b(u, t)) a(u, t-u) r(u) d u,
\end{aligned}
$$

where $b(u, t) \stackrel{\text { def }}{=} \int_{u}^{t} \lambda(s) a(u, s-u) d s$.

Proof. The survival function at time $t$ is given by

$$
\begin{aligned}
S(t) & =E \exp \left(-\int_{0}^{t} h(s) d s\right)=E \exp \left(-\int_{0}^{t} \lambda(s) \int_{0}^{s} a(u, s-u) d Z(R(u)) d s\right) \\
& =E \exp \left(-\int_{0}^{t} b(u, t) d Z(R(u))\right) .
\end{aligned}
$$

Introducing the transformation $v=R(u)$ yields the following form:

$$
S(t)=E \exp \left(-\int_{0}^{R(t)} b\left(R^{-1}(v), t\right) d Z(v)\right)=\exp \left(-\int_{0}^{R(t)} \Phi\left(b\left(R^{-1}(v), t\right)\right) d v\right) .
$$

Transforming back again to $u$ yields the result for $S(t)$. The hazard rate corresponding to this survival function is given by

$$
\begin{aligned}
\mu(t) & =\frac{d}{d t} \int_{0}^{t} \Phi(b(u, t)) r(u) d u=\int_{0}^{t} \frac{d}{d t} \Phi(b(u, t)) r(u) d u \\
& =\lambda(t) \int_{0}^{t} \Phi^{\prime}(b(u, t)) a(u, t-u) r(u) d u .
\end{aligned}
$$

\subsection{Special cases}

Special case 1: Moving average. Let $a(t, v) \equiv a(v)$ depend only on the second argument and let $r(t) \equiv \lambda(t) \equiv 1$ (any constant value of $\lambda$ can be absorbed in $a$, and any constant value of $r$ can be absorbed in $\Phi)$. Since $h(t)=\int_{0}^{t} a(t-u) d Z(u)=$ $\int_{0}^{t} a(v) d Z(t-v), h$ is seen to be a moving average process (although not necessarily stationary). Define $A(v)=\int_{0}^{v} a(u) d u$. Then $b(u, t)=A(t-u)$ and

$$
S(t)=\exp \left(-\int_{0}^{t} \Phi(A(v)) d v\right) \text { and } \mu(t)=\Phi(A(t)) .
$$

Note that $\mu(t)$ is increasing, and in addition concave if $a$ is decreasing. It is clear that if either $\Phi$ is bounded (i.e. $Z$ is compound Poisson,) or $A(\infty) \stackrel{\text { def }}{=} \lim _{t \rightarrow \infty} A(t)<\infty$, then $\lim _{t \rightarrow \infty} \mu(t)=\Phi(A(\infty))<\infty$, so that the hazard converges to a limit, and it 
is reasonable to assume that there is a quasi-stationary distribution for the hazard of survivors. We will later prove this to be true in many cases.

Special case 2: Standard frailty model. We assume $a(t, v) \equiv 1$. Let $r(t)$ be equal to $\rho$ up to time $T$ and 0 after this time, and assume that $\lambda(t)$ is equal to 0 up to time $T$. From the general model (3) it follows that the hazard process equals

$$
h(t)=\lambda(t) Z(\rho T), \quad t \geq 0 .
$$

The population hazard rate is $\mu(t)=\rho T \lambda(t) \Phi^{\prime}(\Lambda(t)), \quad t \geq 0$, where $\Lambda(t) \stackrel{\text { def }}{=} \int_{0}^{t} \lambda(s) d s$. We recognize the hazard rate of the standard frailty model, where the frailty distribution is generated by a Lévy process, as are almost all common frailty distributions. For instance the PVF distributions described in [13] are distributions of Lévy processes, as discussed in Section 6.

Special case 3: Frailty equals instantaneous jump of Lévy process. Assume that $a(t, v)$ depends only on the argument $v$, and that it equals the Dirac delta function in this argument. Then

$$
\mu(t)=r(t) \Phi(\lambda(t)) .
$$

\section{Distribution of frailty for survivors}

In the general frailty model discussed above one may be interested in the conditional distribution of the Lévy process $Z(t)$ generating the frailty given survival up to time $t$, and also the conditional distribution of the frailty $h(t)$ itself. As before, let $T$ be the random time to an event for an individual. We have the following expression for a conditional Laplace functional of the (time transformed) Lévy process $Z(R(t))$ :

Proposition 1. For a suitable non-negative function $c$ on $(0, \infty)$,

$$
\begin{aligned}
L^{\text {surv }}(c ; t) & \stackrel{\text { def }}{=} E\left[\exp \left(-\int_{0}^{\infty} c(s) d Z(R(s))\right) \mid T>t\right] \\
& =\exp \left(-\int_{0}^{\infty}\left(\Phi\left(c(s)+b^{*}(s, t)\right)-\Phi\left(b^{*}(s, t)\right)\right) r(s) d s\right)
\end{aligned}
$$

where $b^{*}(u, t) \stackrel{\text { def }}{=} \mathbb{I}(u \leq t) b(u, t)=\mathbb{I}(u \leq t) \int_{u}^{t} \lambda(s) a(u, s-u) d s$

In particular, the conditional Laplace transform of the hazard $h$ at time $t_{0}$ is

$$
\begin{aligned}
L_{h}^{\text {surv }}\left(c ; t_{0}, t\right) & \stackrel{\text { def }}{=} E\left[e^{-c h\left(t_{0}\right)} \mid T>t\right] \\
& =\exp \left\{-\int_{0}^{t_{0}}\left[\Phi\left(c \lambda\left(t_{0}\right) a\left(s, t_{0}-s\right)+b^{*}(s, t)\right)-\Phi\left(b^{*}(s, t)\right)\right] r(s) d s\right\},
\end{aligned}
$$

and

$$
\begin{aligned}
E\left[h\left(t_{0}\right) \mid T>t\right] & =\lambda\left(t_{0}\right) \int_{0}^{t_{0}} \Phi^{\prime}\left(b^{*}(s, t)\right) a\left(s, t_{0}-s\right) r(s) d s, \\
\operatorname{Var}\left[h\left(t_{0}\right) \mid T>t\right] & =-\lambda\left(t_{0}\right)^{2} \int_{0}^{t_{0}} \Phi^{\prime \prime}\left(b^{*}(s, t)\right) a\left(s, t_{0}-s\right)^{2} r(s) d s .
\end{aligned}
$$


Proof. Let as before $\mathbb{I}$ be an indicator function.

$$
\begin{aligned}
L^{\text {surv }}(c ; t) & =\mathrm{E}\left[\exp \left\{-\int_{0}^{\infty} c(s) d Z(R(s))\right\} \mid T>t\right] \\
& =\mathrm{E}\left[\exp \left\{-\int_{0}^{\infty} c(s) d Z(R(s))\right\} \mathbb{I}(T>t)\right] / P(T>t) \\
& =\mathrm{E}\left[\exp \left\{-\int_{0}^{\infty} c(s) d Z(R(s))\right\} E[\mathbb{I}(T>t) \mid Z]\right] S_{t}^{-1} \\
& =\mathrm{E}\left[\exp \left\{-\int_{0}^{\infty}\left(c(s)+b^{*}(s, t)\right) d Z(R(s))\right\}\right] S_{t}^{-1} \\
& =\exp \left\{-\int_{0}^{\infty}\left(\Phi\left(c(s)+b^{*}(s, t)\right)-\Phi\left(b^{*}(s, t)\right)\right) r(s) d s\right\}
\end{aligned}
$$

The expression for $L_{h}^{\text {surv }}\left(c ; t_{0}, t\right)$ follows by letting $c(s)=c \mathbb{I}\left(s \leq t_{0}\right) \lambda\left(t_{0}\right) a\left(s, t_{0}-s\right)$, and the conditional expectation and variance for $h\left(t_{0}\right)$ follows by differentiation of $L_{h}^{\text {surv }}$.

Remark 1. In the above Proposition, a typical function $c(s)$ would be a step function having constant values on intervals and being zero after a certain point. This will produce the joint Laplace transform of increments of the $Z \circ R$ process, conditional on no event. For instance, it follows that the distribution of the increment $Z\left(R\left(u_{2}\right)\right)-$ $Z\left(R\left(u_{1}\right)\right)$, conditional on $T>t$, has the Laplace transform

$$
\begin{aligned}
E \exp \left(-c\left[Z\left(R\left(u_{2}\right)\right)\right.\right. & \left.\left.-Z\left(R\left(u_{1}\right)\right)\right] \mid T>t\right) \\
& =\exp \left(-\int_{u_{1}}^{u_{2}}\left(\Phi\left(c+b^{*}(s, t)\right)-\Phi\left(b^{*}(s, t)\right)\right) r(s) d s\right) \\
& =\exp \left(-c \AA\left(R\left(u_{2}\right)-R\left(u_{1}\right)\right)-\int_{(0, \infty)}\left(1-e^{-c x}\right) \Pi_{u_{1}, u_{2}, t}(d x)\right)
\end{aligned}
$$

where

$$
\Pi_{u_{1}, u_{2}, t}(d x)=\left\{\int_{u_{1}}^{u_{2}} e^{-b^{*}(s, t) x} r(s) d s\right\} \Pi(d x) .
$$

Thus, for fixed $u_{1}, u_{2}, t$, the increment $Z\left(R\left(u_{2}\right)\right)-Z\left(R\left(u_{1}\right)\right)$ has the distribution of $\tilde{Z}(1)$, a Lévy process with drift $₫\left(R\left(u_{2}\right)-R\left(u_{1}\right)\right)$ and Lévy measure $\Pi_{u_{1}, u_{2}, t}$. By computations similar to the proof of the proposition, it can be shown that, conditional on $T>t$, the increments of $Z \circ R$ are independent. Similarly, the Laplace transform $L_{h}^{\text {surv }}$ can also be rewritten as the transform of a Lévy process distribution.

For an ordinary Laplace transform $L(c)$ corresponding to a density $f(x)$, the transformation $L(c+b) / L(b)$ for some constant $b$ corresponds to the modified probability density $e^{-b x} f(x) / L(b)$. A similar interpretation would hold above.

Note that the formula (9) closely resembles formula (11) in Barndorff-Nielsen and Shepherd [7]. Their expression is the case where the integrated hazard process $H(t)=$ $\int^{t} h(s) d s$ is modelled directly as

$$
H(t)=\exp (-\lambda t) H(0)+\int_{0}^{t} \exp (-\lambda(t-s)) d Z(\lambda s)
$$

instead of modelling $h(t)$. 
Special case 1 (continued). When $a(t, v) \equiv a(v)$ depends only on the second argument and $\lambda \equiv r \equiv 1$, the variance at time $t$ for the hazard of survivors, with $A(v)=\int_{0}^{v} a(u) d u$ as before, is

$$
\operatorname{Var}[h(t) \mid T>t]=-\int_{0}^{t} \Phi^{\prime \prime}(A(s)) a^{2}(s) d s .
$$

Clearly, $\operatorname{Var}[h(t) \mid T>t]$ is increasing in $t$. We note that if $0<k_{1}<a(s)<k_{2}<\infty$ on some interval $s \in[0, \epsilon)$, then it can be shown that

$$
E Z(t)<\infty \Leftrightarrow \operatorname{Var}[h(t) \mid T>t]<\infty, \quad t \geq 0 .
$$

In particular, let us consider $a(v)=a e^{-\kappa v}$. Then $A(v)=a\left(1-e^{-\kappa v}\right) / \kappa$, and using integration by part,

$$
\begin{aligned}
\mu(t) & =\Phi(A(t)) \\
\operatorname{Var}[h(t) \mid T>t] & =a \Phi^{\prime}(0)-a(t) \Phi^{\prime}(A(t))-\kappa \Phi(A(t)) \\
& =\mu^{\prime}(0)-\mu^{\prime}(t)-\kappa \mu(t) .
\end{aligned}
$$

Similarly, if $a(v) \equiv a$ (constant), then

$$
\mu(t)=\Phi(a t) \text { and } \operatorname{Var}[h(t) \mid T>t]=\mu^{\prime}(0)-\mu^{\prime}(t) .
$$

\section{Long-run behavior and quasi-stationary distributions}

As discussed in the introduction, it is of conceptual and practical importance to decide whether a model may produce an almost constant hazard rate even though the individual hazards may be increasing. In our context, we can ask whether the population hazard $\mu(t)$ converges to a finite value. Also, we are interested in seeing whether the distribution of $h(t)$ or $Z(t)$, conditional on $T>t$, stabilizes to a specific quasi-stationary distribution as $t \rightarrow \infty$. We will prove that this happens under some common circumstances.

Condition 2. The limits $\lambda(\infty), r(\infty)$ and $a(\infty, v)$ of respectively $\lambda(t), r(t)$ and $a(t, v)$ as $t \rightarrow \infty$ all exist and are finite.

Condition 3. $E[Z(1)]<\infty$ and $a(t, v) \leq \tilde{a}(v)$ for some function $\tilde{a}$ with $\int_{0}^{\infty} \tilde{a}(s) d s<$ $\infty$.

Condition 4. There is a $k_{1}$ such that $\lambda(t) \geq k_{1}>0, \forall t \geq 0$. There is an $\epsilon>0$ and a function a such that $\epsilon \tilde{a}(v) \leq a(t, v) \leq \tilde{a}(v)$, and either $\int_{0}^{\infty} \tilde{a}(v) d v<\infty$ or $\Phi$ is bounded (compound Poisson case).

\section{Theorem 2.}

Limiting hazard:Assume Condition 2 holds. If in addition either Condition 3 or Condition 4 holds, the population hazard converges to the finite value

$$
\mu(\infty)=\lim _{t \rightarrow \infty} \mu(t)=r(\infty) \Phi(\eta(\infty))
$$

where $\eta(v) \stackrel{\text { def }}{=} \lambda(\infty) \int_{0}^{v} a(\infty, s) d s$.

Quasi-stationary distribution:Under the same conditions, a quasi-stationary distribution exists for $h(t)$, conditional on $T>t$, as $t \rightarrow \infty$. The Laplace transform of the 
quasi-stationary distribution is

$$
\begin{aligned}
L_{h}^{\text {surv }}(c ; \infty) & =\lim _{t \rightarrow \infty} L_{h}^{\text {surv }}(c ; t, t)=\exp \left\{-r(\infty) \int_{0}^{\infty}\left[\Phi\left(c \eta^{\prime}(v)+\eta(v)\right)-\Phi(\eta(v))\right] d v\right\} \\
& =\exp \left\{-\left[d r(\infty) \eta(\infty) c+\int_{(0, \infty)}\left(1-e^{-c z}\right) \tilde{\Pi}(d z)\right]\right\} .
\end{aligned}
$$

Here $\tilde{\Pi}$ is a new Lévy measure defined by

$$
\tilde{\Pi}(d z)=r(\infty) \int_{\left\{v: \eta^{\prime}(v)>0\right\}} \exp \left(-\frac{\eta(v)}{\eta^{\prime}(v)} z\right) \Pi\left(\frac{d z}{\eta^{\prime}(v)}\right) d v
$$

$₫$ and $\Pi$ are the drift coefficient and Lévy measure for $\Phi$. Thus, the quasi-stationary distribution is the distribution of a random variable $\tilde{Z}(1)$, where $\tilde{Z}$ is a new subordinator with drift $r(\infty) d \eta(\infty)$ and Lévy measure $\tilde{\Pi}$.

Proof. Limiting hazard: After a change of variable we get

$$
\mu(t)=\lambda(t) \int_{0}^{\infty} \mathbb{I}(v \leq t) \Phi^{\prime}(b(t-v, t)) a(t-v, v) r(t-v) d v
$$

Under Condition 3 we see that $\Phi^{\prime}(0)=E[Z(1)]<\infty$, and since $\Phi^{\prime}$ is decreasing, the integrand is dominated by $E[Z(1)] \sup _{t \geq 0} r(t) \tilde{a}(v)$, which is integrable. The result follows from the dominated convergence theorem by taking the limit under the integral sign.

Under Condition 4 we notice that $b(t-v, t) \geq k_{1} \epsilon \int_{0}^{v} \tilde{a}(s) d s$ and consequently the integrand is dominated by $\Phi^{\prime}\left(k_{1} \epsilon \int_{0}^{v} \tilde{a}(s) d s\right) \tilde{a}(v) \sup _{t>0} r(t)$. By the chain rule, this will have a finite integral over $[0, \infty)$ provided either $\bar{\Phi}$ is bounded or $\tilde{a}$ is integrable. The result again follows by the dominated convergence theorem.

Quasi-stationary distribution: After a change of variable,

$$
\begin{aligned}
L_{h}^{\text {surv }}(c ; t, t)= & \exp \left\{-\int_{0}^{\infty} \mathbb{I}(v \leq t)[\Phi(c \lambda(t) a(t-v, v)+b(t-v, t))\right. \\
& -\Phi(b(t-v, t))] r(t-v) d v\} .
\end{aligned}
$$

By the concavity of $\Phi$ we have

$$
\Phi(c \lambda(t) a(t-v, v)+b(t-v, t))-\Phi(b(t-v, t)) \leq \Phi^{\prime}(b(t-v, t)) c \lambda(t) a(t-v, v)
$$

By an argument identical to the one above,

$$
\begin{aligned}
\lim _{t \rightarrow \infty} L_{h}^{\operatorname{surv}}(c ; t, t)= & \exp \left\{-r(\infty) \int_{0}^{\infty}\left[\Phi\left(c \eta^{\prime}(v)+\eta(v)\right)-\Phi(\eta(v))\right] d v\right\} \\
= & \exp \{-\mathrm{d} r(\infty) \eta(\infty) c \\
& \left.-r(\infty) \int_{0}^{\infty} \int_{(0, \infty)} e^{-\eta(v) x}\left(1-e^{-c \eta^{\prime}(v) x}\right) \Pi(d x) d v\right\} \\
= & \exp \left[-\left[\mathrm{d} r(\infty) \eta(\infty) c+\int_{(0, \infty)}\left(1-e^{-c z}\right) \tilde{\Pi}(d z)\right]\right],
\end{aligned}
$$


where the last part follows after a change of variable from $x$ to $z=\eta^{\prime}(v) x$. It remains to be shown that $L_{h}^{\text {surv }}(c ; \infty)$ is continuous in $c=0$. But this follows from

$$
\int_{0}^{\infty}\left[\Phi\left(c \eta^{\prime}(v)+\eta(v)\right)-\Phi(\eta(v))\right] d v \leq c \int_{0}^{\infty} \Phi^{\prime}(\eta(v)) \eta^{\prime}(v) d v=c \Phi(\eta(\infty)),
$$

and thus $L_{h}^{\text {surv }}(c ; \infty)$ is a proper Laplace transform of a quasi-stationary distribution.

Remark 2. For PVF processes we have $E[Z(1)]=\Phi^{\prime}(0)=n \rho / \nu<\infty$, so that the first part of Condition 3 is satisfied.

Remark 3. Letting $c \rightarrow \infty$ in (2), we have

$$
P(Z(t)=0)=\exp (-t \Phi(\infty)) .
$$

Thus, in the common situation covered by Condition 4 , with $Z$ compound Poisson but $\eta(\infty)=\infty$, it is clear that $\mu(t)$ stabilizes because of a subgroup of individuals for whom $Z(t)=0$, and $\Phi(\infty)$ is the rate at which these individuals are released through a "bottleneck" from the no risk group into a group with higher risk.

Remark 4. Quasi-stationary distribution for $Z(R(t))$. It is also relevant to ask whether the underlying process $Z \circ R$ reaches quasi-stationarity. The conditions on $a$ for this to be true are somewhat different from the $h$ case. Together with Condition 2, it is sufficient to have $\Phi$ bounded, and that there exist constants $k_{1}, l>0$ such that $\lambda(t) \geq k_{1}>0$ and $a(t, v) \geq l$, for all $t, v \geq 0$. Under these conditions, there is a quasi-stationary distribution for $Z(R(t))$ conditional on $T>t$, with Laplace transform

$\lim _{t \rightarrow \infty} E[\exp \{-c Z(R(t))\} \mid T>t]=\exp \left\{-\left[c r(\infty) \mathrm{d} \eta(\infty)+\int_{(0, \infty)}\left(1-e^{-c z}\right) \tilde{\Pi}(d z)\right]\right\}$

where $\tilde{\Pi}(d z)=r(\infty) \int_{0}^{\infty} e^{-\eta(v) z} d v \Pi(d z)$.

Special case 1 (continued). When $a(t, v) \equiv a(v)$ and $\lambda \equiv r \equiv 1$, we have already mentioned that $\mu(t)=\Phi(A(t))$ is increasing, and has a limit $\Phi(A(\infty))$ exactly when $\Phi$ is bounded (the compound Poisson case) or when $A$ is bounded.

Similarly, for the variance we have seen that $\operatorname{Var}[h(t) \mid T>t]=-\int_{0}^{t} \Phi^{\prime \prime}(A(s)) a^{2}(s) d s$ is increasing, and under Condition 3 it is true that

$$
\lim _{t \rightarrow \infty} \operatorname{Var}[h(t) \mid T>t]=-\int_{0}^{\infty} \Phi^{\prime \prime}(A(s)) a^{2}(s) d s<\infty .
$$

Specifically, if $a(v)=a e^{-\kappa v}$ then $\mu(\infty)=\Phi(a / \kappa)$ and

$$
\lim _{t \rightarrow \infty} \operatorname{Var}[h(t) \mid T>t]=a E[Z(1)]-\kappa \mu(\infty) .
$$

If $a(v)$ is constant in $v$, then $\mu(\infty)=\Phi(\infty)$ and

$$
\lim _{t \rightarrow \infty} \operatorname{Var}[h(t) \mid T>t]=a E[Z(1)] .
$$

Furthermore, in this case we also have a simple expression for the Lévy measure of the limiting distribution of $h$, following Theorem 2:

$$
\tilde{\Pi}(d z)=\int_{0}^{\infty} e^{-v z} \Pi\left(\frac{d z}{a}\right) d v=\frac{1}{z} \Pi\left(\frac{d z}{a}\right),
$$


which at least in the compound Poisson case defines a proper quasi-stationary distribution.

\section{PVF processes}

Recall that a PVF Lévy process is determined by the Laplace exponent

$$
\Phi_{\mathrm{PVF}}(c ; \rho, \nu, n)=\rho\left\{1-\left(\frac{\nu}{\nu+c}\right)^{n}\right\} .
$$

In the case of PVF processes, many of the formulae given in the preceding results can be made explicit in particular cases. We will focus on situations where $\lambda(t)=t^{k}$ for some $k \geq 0, r(t) \equiv 1$ and $a(t, v) \equiv a$ (constant). That is, the frailty process can be written as

$$
h(t)=a t^{k} Z(t)
$$

so that the hazard consists of a deterministic Weibull part, perturbed by a Lévy process. For various combinations of values of $k$ and $n$ we can now study the properties of the population hazard $\mu$ and the distribution of survivors. Notice first that

$$
E[h(t)]=a m t^{k+1} \text { and } \operatorname{Var}[h(t)]=a^{2} \tau^{2} t^{2 k+1}
$$

with $m=E[Z(1)]=n \rho / \nu$ and $\tau^{2}=\operatorname{Var}[Z(1)]=n(n+1) \rho / \nu^{2}$. Clearly, $h(t)$ is an increasing process with $\operatorname{Var}[h(t)] \rightarrow \infty$ as $t \rightarrow \infty$.

\subsection{The distribution of survivors at time $t$}

The population hazard rate is

$$
\mu(t)=n \rho(k+1) \gamma(t)^{-n}\left(\frac{\gamma(t)-1}{\gamma(t)}\right) H\left(\frac{1}{k+1}, n+1, \frac{k+2}{k+1}, \frac{\gamma(t)-1}{\gamma(t)}\right)
$$

with

$$
\gamma(t) \stackrel{\text { def }}{=} 1+\frac{a}{\nu(k+1)} t^{k+1},
$$

where $H$ denotes the hypergeometric function [15]. To compute $\mu$ we used Mathematica (Wolfram Research, Inc.) to integrate formula (8). Clearly, as a parametric model, one of the parameters $a, \nu$ is redundant. From the definition (3) of $h(t)$ it can be seen that in general, the value of $\nu$ can be absorbed into a multiplicative constant in $a$. Thus, henceforth we will let $\nu=1$ in our PVF examples.

Considering the variance of the hazard of survivors, the following expression can be derived:

$$
\begin{aligned}
\operatorname{Var}[h(t) \mid T>t]= & n(n+1) \rho(k+1)^{2} \frac{1}{t} \gamma(t)^{-n} \\
& \times\left(\frac{\gamma(t)-1}{\gamma(t)}\right)^{2} H\left(\frac{1}{k+1}, n+2, \frac{k+2}{k+1}, \frac{\gamma(t)-1}{\gamma(t)}\right) .
\end{aligned}
$$

Notice the similarity to $\mu(t)$. Similarly, the conditional Laplace transform can be 
computed:

$$
\begin{aligned}
L_{h}^{\text {surv }}(c ; t)= & E\left[e^{-c h(t)} \mid T>t\right] \\
= & \exp \left[-\rho t\left\{\gamma(t)^{-n} H\left(\frac{1}{k+1}, n, \frac{k+2}{k+1}, \frac{\gamma(t)-1}{\gamma(t)}\right)\right.\right. \\
& \left.\left.-\left(\gamma(t)+c a t^{k}\right)^{-n} H\left(\frac{1}{k+1}, n, \frac{k+2}{k+1}, \frac{\gamma(t)-1}{\gamma(t)+c^{k} t^{k}}\right)\right\}\right] .
\end{aligned}
$$

\subsection{Behavior when $t \rightarrow \infty$}

Using asymptotic properties of the hypergeometric functions ([15]), we can show that in the compound Poisson case $n>0, \mu(t) \rightarrow \rho$ as $t \rightarrow \infty$. Otherwise, when $0>n>-1$ we have

$$
\mu(t) \sim n \rho\left(\frac{k+1}{a}\right)^{n} B\left(\frac{1}{k+1},-n\right) t^{-n(k+1)} \rightarrow \infty \text { as } t \rightarrow \infty .
$$

Here, $B(x, y)=\int_{0}^{1} t^{x-1}(1-t)^{y-1} d t$ is the beta function. For the variance we find the asymptotic relation

$$
\operatorname{Var}[h(t) \mid T>t] \sim a n \rho t^{k}, \quad t \rightarrow \infty .
$$

We see that the population behavior is markedly different from the individual hazards. In the compound Poisson case we reach a stable value for $\mu(t)$ even when the individual hazards are increasing. However, only in the case where $k=0$ will the variance stabilize around a constant value over time (regardless of $n$ ), creating a quasi-stationary distribution.

The upper panel in Figure 1 illustrates the behavior of the population hazard. All hazards are computed using the values $a=1 / 2$ and $k=2$, but with varying values of $n$ and $\rho$. The two hazards when $(n, \rho)$ equals $(-0.5,-5)$ or $(1,5)$ are quite different. However, the two corresponding density functions (lower panel) are almost identical. This is due to the fact that the two hazards are fairly similar up to time 2, and within that time almost all of the population has had an event. As a consequence, convergence of $\mu$ is irrelevant in this case. A different situation emerges when $(n, \rho)$ equals $(-0.5,-0.25)$ or $(1,0.25)$. Although the hazards are identical in shape to the previous two, the overall hazard level is much lower and events accumulate much more slowly. The result is that the one hazard approaches the steady state and the other goes to infinity long before all individuals have had an event, producing two very different densities. Clearly, convergence of $\mu$ is here important for the life time distribution.

\subsection{The particular case $k=0$}

When $k=0$ we simply have $h(t)=a Z(t)$, and the results are also covered by Special case 1 . We have

$$
\mu(t)=\Phi_{\mathrm{PVF}}(A(t) ; \rho, \nu, n)=\Phi_{\mathrm{PVF}}(a t ; \rho, 1, n)=\Phi_{\mathrm{PVF}}(t ; \rho, 1 / a, n)
$$

and

$$
\operatorname{Var}[h(t) \mid T>t]=a \Phi_{\mathrm{PVF}}^{\prime}(0 ; \rho, \nu, n)-\mu^{\prime}(t)=\Phi_{\mathrm{PVF}}(t ; a n \rho, 1 / a, n+1) .
$$



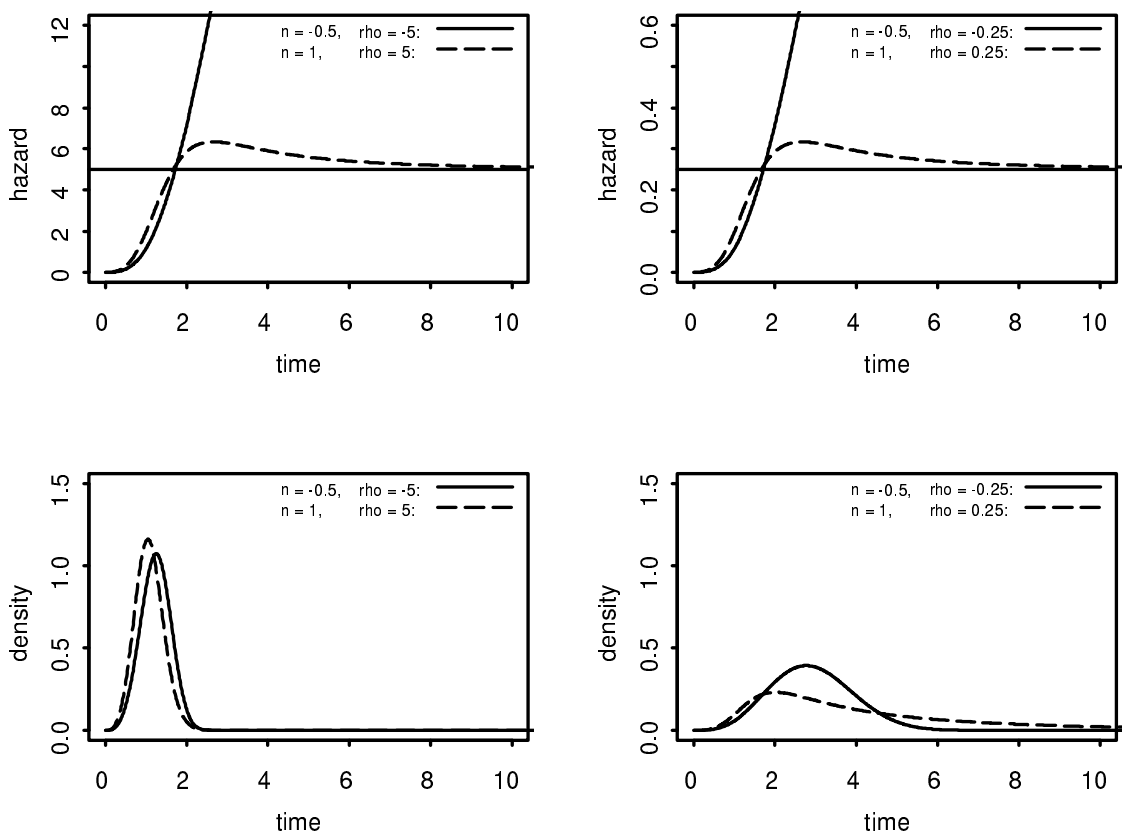

FiguRE 1: Hazard rate (upper row) and their corresponding life time densities (lower row). Generated by $h(t)=\frac{1}{2} t^{2} Z(t)$, where $Z(t)$ is PVF with $\nu=1$ but varying values of $n$ and $\rho$.

The Laplace transform simplifies to

$$
\begin{aligned}
L_{h}^{\text {surv }}(c ; t)= & \exp \left[-\frac{\rho}{a(n-1)}\left\{1+\left(\frac{1}{1+a(t+c)}\right)^{n-1}-\left(\frac{1}{1+a c}\right)^{n-1}-\left(\frac{1}{1+a t}\right)^{n-1}\right\}\right] \\
= & \exp \left[-\Phi_{\mathrm{PVF}}\left(c ; \frac{\rho}{a(n-1)}, 1 / a, n-1\right)\right] \\
& / \exp \left[-\Phi_{\mathrm{PVF}}\left(c ; \frac{\rho}{a(n-1)}\left(\frac{1}{1+a t}\right)^{n-1}, 1 / a+t, n-1\right)\right] .
\end{aligned}
$$

If $n>0$, this is a quotient between two PVF Laplace transforms. The expectations of the PVF distributions corresponding to the two Laplace transforms are

$$
\rho \text { and } \rho\left(\frac{1}{1+a t}\right)^{n}
$$

respectively, while the variances are

$$
\text { an } \rho \text { and } \operatorname{an} \rho\left(\frac{1}{1+a t}\right)^{n-1} \text {. }
$$

Asymptotically, as $t \rightarrow \infty$,

$$
\begin{aligned}
& \mu(t) \quad \rightarrow \quad \rho \text { when } n>0 \\
& \mu(t) \sim-\rho(a t)^{-n} \rightarrow \infty \text { when } 0>n>-1,
\end{aligned}
$$


and

$$
\operatorname{Var}[h(t) \mid T>t] \rightarrow \text { an } \rho
$$

regardless of $n$. The limiting distribution has the Lévy measure (see Special case 1)

$$
\tilde{\Pi}(d z)=n \rho \frac{1}{a^{n} \Gamma(n+1)} z^{n-2} e^{-z / a} d z,
$$

which defines a proper quasi-stationary distribution when $n>0$, and this is a PVF distribution with Laplace exponent $\Phi_{\mathrm{PVF}}(c ; \rho /(a(n-1)), 1 / a, n-1)$.

\section{Random perturbations of hazard functions}

In this section we use results reached above to investigate some natural hazard rate models that come from placing random frailty processes "around" given deterministic hazard functions. Using such frameworks of varying frailty we learn more about the consequences for the hazard rates that can actually be observed, i.e. when estimated from individuals sampled from a population. It is a basic lesson of ordinary fixed-frailty theory that the shape of the population hazard may be quite different from that of each individual; this phenomenon is partly even more pronounced in our context of frailty processes, since the mechanisms are more complex.

Assume first that the hazard rate, conditional on a multiplicative frailty variable $F$, takes the form $h_{0}(t)=\lambda(t) F$, where the $\lambda$ function is fixed. For a random life time $T$ this implies $P[T \geq t \mid F]=\exp [-\Lambda(t) F]$ in terms of the cumulative base hazard function $\Lambda(t)=\int_{0}^{t} \lambda(s) d s$. If $F$ has Laplace transform $E \exp (-c F)=\exp \{-G(c)\}$, say, then the unconditional survivor function is $P(T \geq t)=\exp \{-G(\Lambda(t))\}$. This is the classic multiplicative frailty situation, with ensuing hazard rate $\mu(t)=G^{\prime}(\Lambda(t)) \lambda(t)$ for individuals sampled from the population; see e.g. [13], Ch. 2. Among the perhaps too strict implications of this frailty formulation is that $h_{0}(t)$ differs from $\lambda(t)$ by a fixed factor for all $t$, in particular the correlation between $h_{0}(s)$ and $h_{0}(t)$ is 1 for all $s$ and $t$. Also, the multiplicative correction to the basis hazard $\lambda(t)$ implied by the random frailty $F$ takes the very precise form $\rho(t)=G^{\prime}(\Lambda(t))$, whereas other and more flexible forms of correction might be anticipated for various applications. In particular, this classic formulation entails by necessity a monotone decreasing $\rho(t)$. Our model (3) is much more flexible in these regards.

Write

$$
a(u, t-u)=\frac{a_{0}(u, t-u)}{m A_{0}(t)},
$$

where $A_{0}(t) \stackrel{\text { def }}{=} \int_{0}^{t} a_{0}(u, t-u) r(u) d u$, for some appropriate function $a_{0}(t, v)$. Let $m=E[Z(1)]$ and $\tau^{2}=\operatorname{Var}[Z(1)]$, assuming these quantities exist. Using this definition of $a$ in (3) we can write

$$
h(t)=\lambda(t) F(t)
$$

where

$$
F(t)=\frac{1}{m A_{0}(t)} \int_{0}^{t} a_{0}(u, t-u) d Z(R(u)) .
$$

From (6) it is clear that $E[F(t)]=1$, thus $F(t)$ can be thought of as a random perturbation of the deterministic hazard process $\lambda$. From (7) we have

$$
V(t) \stackrel{\text { def }}{=} \operatorname{Var}[F(t)]=\frac{\tau^{2}}{m^{2} A_{0}(t)^{2}} \int_{0}^{t} a_{0}(u, t-u)^{2} r(u) d u .
$$


One also finds that the correlation between $h(s)$ and $h(t)$ is $\{V(s) / V(t)\}^{1 / 2}$ for $s \leq t$.

In the rest of the section we will assume $r(t) \equiv 1$. Some examples illustrate different strands of behavior for the frailty process $F(t)$.

(i) If $a_{0}$ is constant then $h(t)=\lambda(t) Z(t) /(m t)$ and $V(t)=\left(\tau^{2} / m^{2}\right) / t$, which means that $F(t)$ starts out highly variable but settles down towards the value 1 as time goes on. Also, the correlation between $h(s)$ and $h(t)$ is $(s / t)^{1 / 2}$ for $s \leq t$, indicating that $h(t)$ 'forgets' $h(s)$ as $t$ moves on. One finds similar results for $a(u, t-u)$ equal to either $u^{k}$ or $(t-u)^{k}$, for positive parameters $k$.

(ii) The two situations $a_{1}(u, t-u)=\exp (-\kappa u)$ and $a_{2}(u, t-u)=\exp (-\kappa(t-$ $u)$ ), where $\kappa$ is a positive smoothing parameter, correspond to rather different frailty processes, in that the first places most emphasis on the start of the $Z(v)$ process, i.e. for small $v$, while the second has $F(t)$ more dependent on recent $Z(v)$ behavior, i.e. for $v$ closer to $t$. Both models have the same variance and correlation structure, however, with

$$
V(t)=\frac{\tau^{2}}{m^{2}} \frac{1}{2} \kappa \frac{1-\exp (-2 \kappa t)}{\{1-\exp (-\kappa t)\}^{2}} .
$$

This is proportional to $1 / t$ for $t$ small but goes towards a fixed positive limit when $t$ grows, i.e. the frailty process will not stop exhibiting real variation. There is also a long-term memory aspect of this situation.

(iii) One may similarly investigate $a(u, t-u)$ equal to $\exp (\kappa u)$ or $\exp (\kappa(t-u))$. We find the same expression for $V(t)$ as for case (ii).

By (8), the population hazard rate is

$$
\mu(t)=\lambda(t) \rho(t)
$$

where

$$
\rho(t)=\frac{1}{m A_{0}(t)} \int_{0}^{t} \Phi^{\prime}(b(u, t)) a_{0}(u, t-u) d u
$$

and

$$
b(u, t)=\int_{u}^{t} \frac{\lambda(s)}{m A_{0}(s)} a_{0}(u, s-u) d s .
$$

We may read this as having the basis hazard rate $\lambda(t)$ modified with a correction function $\rho(t)$ due to the extra frailty variation in the population, causing early deaths among those with higher frailty. There is a variety of shapes and types of the $\rho$ function, depending not only on $\lambda$ but also on the weight function $a$ and the Laplace exponent $\Phi$. Note that $\rho(t)$ is always less than 1 .

The simplest special case here is that of $a_{0}$ being constant, in which case $\rho(t)=$ $(m t)^{-1} \int_{0}^{t} \Phi^{\prime}(b(u, t)) d u$, where $b(u, t)=m^{-1} \int_{u}^{t} s^{-1} \lambda(s) d s$. When $\lambda$ is constant, this simply gives a constant population hazard rate $\mu(t)=\lambda \rho$, since the frailty correction function $\rho(t)$ is found to be equal to the constant $\rho=m^{-1} \int_{0}^{\infty} \Phi^{\prime}(\lambda v / m) \exp (-v) d v$. This differs from classical applications of frailty, where the shape of the population hazard invariably is different from that of the individuals.

Next look at Weibull base shapes $\lambda(t)=\lambda t^{k}$, excepting the case $k=0$ just dealt with. Here

$$
\mu(t)=\lambda(t) \frac{1}{m t} \int_{0}^{t} \Phi^{\prime}\left(\frac{\lambda}{m} k^{-1}\left(t^{k}-u^{k}\right)\right) d u .
$$


Choosing different values of $k$ and $\lambda$ reveals a range of different shapes for the correction function $\rho(t)$. It is for example possible to have the population $\mu(t)$ reasonably flat, coming from an increasing $\lambda(t)$ for each individual, but counterbalanced with the frailty mechanism.

We also learn from the special type of $\lambda(t)=t q(t)$, say, where $q$ has integral function $Q$, that

$$
\mu(t)=\lambda(t) \frac{1}{m t} \int_{0}^{t} \Phi^{\prime}\left(m^{-1}(Q(t)-Q(u))\right) d u .
$$

For $\lambda(t)=\lambda_{0} t \exp (-k t)$, for example, one finds correction curves that first decrease and then increase, causing sometimes different shapes for $\mu(t)$ than for each individual's basic $\lambda(t)$. Specifically, the peak of maximum individual hazard is pushed downwards in size, and happens earlier when observed in a random sample.

Further examples of interest are afforded by the two cases $a_{0}^{(1)}(u, t-u)=\exp (-\kappa u)$ and $a_{0}^{(2)}(u, t-u)=\exp (-\kappa(t-u))$, where $\kappa$ again is a positive smoothing parameter. For these cases, coupled with a constant $\lambda$, we find

$$
\begin{aligned}
\rho^{(1)}(t)= & \frac{\kappa / m}{1-\exp (-\kappa t)} \int_{0}^{t} \Phi^{\prime}\left(\frac{\lambda}{m} \exp (-\kappa u) \log \frac{\exp (\kappa t)-1}{\exp (\kappa u)-1}\right) \exp (-\kappa u) d u, \\
\rho_{(2)}(t)= & \frac{\kappa / m}{1-\exp (-\kappa t)} \\
& \times \int_{0}^{t} \Phi^{\prime}\left(\frac{\lambda}{m} \exp (\kappa u)\left\{\log \frac{\exp (\kappa t)-1}{\exp (\kappa u)-1}-\kappa(t-u)\right\}\right) \exp (-\kappa(t-u)) d u
\end{aligned}
$$

Often, the second correction curve decreases more rapidly towards zero, the first retaining more from the values at the start. Also, the $\rho_{2}(t)$ function may be first increasing before decreasing. This also happens with other $\lambda(t)$ functions.

\section{References}

[1] Aalen, O. O. (1992). Modelling heterogeneity in survival analysis by the compound Poisson distribution. Annals of Applied Probability 2 951-972

[2] Aalen, O. O. (1994). Effects of frailty in survival analysis. Statistical Methods in Medical Research 3 227-243

[3] Aalen, O. O. and Guessing, H. K. (2001). Understanding the shape of the hazard rate: A process point of view. Statistical Science 16, No. 1, 1-22

[4] Aalen, O. O. and HJoRT, N. L. (2002). Frailty models that yield proportional hazards. Statistics 83 Probability Letters 58 335-342

[5] Aalen, O. O. and Tretli, S. (1999). Analyzing incidence of testis cancer by means of a frailty model. Cancer Causes and Control 10 285-292

[6] Andersen, P.K., Borgan, Ø., Gill, R. and Keiding, N. (1994). Statistical Models Based on Counting Processes. Springer, New York.

[7] Barndorff-Nielsen, O.E. And Shephard, N. (2001). Non-Gaussian Ornstein-Uhlenbeck-based models and some of their uses in financial economics. J.R. Statist. Soc. B 63, Part 2, 167-241 (With discussion) 
[8] Bertoin, J. (1996). Lévy Processes. Cambridge University Press, Cambridge.

[9] Clarke, C., Collins, R. A., Leavitt, B. R., Andrews, D. F., Hayden, M. R., Lumsden, C. J. AND MCINNES, R. R. (2000). A one-hit model of cell death in inherited neuronal degenerations. Nature 406 195-199

[10] Clarke, C., Lumsden, C. J. And McInnes, R. R. (2001). Inherited neurodegenerative diseases: the one-hit model of neurodegeneration. Human Molecular Genetics 10 2269-2275

[11] HJoRT, N. L. (2003). Topics in non-parametric Bayesian statistics [with discussion]. In Highly Structured Stochastic Systems (eds. P.J. Green, N.L.Hjort and S. Richardson), Oxford University Press (to appear).

[12] Hougand, P. (1986). Survival models for heterogeneous populations derived from stable distributions. Biometrika $\mathbf{7 3}$ 387-396. (Correction ibid. 75 395)

[13] Houga ARD, P. (2000). Analysis of Multivariate Survival Data. Springer, New York.

[14] KeBiR, Y. (1991). On hazard rate processes. Naval Research Logistics 38 865-876

[15] Lebedev, N. N. (1972). Special Functions and Their Applications. Dover Publications, INC., New York.

[16] Lo, C.-C., Nunes Amaral, L. A., Havlin, S., Ivanov, P. Ch., Penzel, T., Peter, J.-H. and StAnLey, H. E. (2002). Dynamics of sleep-wake transitions during sleep. Europhysics Letters $\mathbf{5 7}$ $625-631$

[17] SingpurWalla, N. D. (1995). Survival in dynamic environments. Statistical Science 10, No. 1, $86-103$

[18] Slate, E. H. and Turnbull, B. W. (2000). Statistical models for longitudinal biomarkers of disease onset. Statistics in Medicine 19,617-637

[19] Triarhou, L. C. (1998). Rate of neuronal fallout in a transsynaptic cerebellar model. Brain Research Bulletin 47 219-222

[20] Yashin, A.I. and Manton, K.G. (1997). Effects of unobserved and partially observed covariate processes on system failure: A review of models and estimation strategies. Statistical Science 12, No. $1,20-34$ 